\title{
Multiresolution molecular mechanics: A unified and consistent framework for general finite element shape functions
}

\author{
Yang, Qingcheng; To, Albert, University of Pittsburgh, United States
}

\begin{abstract}
We present a general mathmatical framework for the newly proposed energy-based concurrent atomistic/continuum method Multiresolution Molecular Mechanics (MMM) The main features of the generalized framework are: (i) Consistency with the atomistic framework by directly employing the interatomic potential to calculate force and energy; (ii) Simple procedure for analytically deriving the optimal summation rule for any given finite element shape function employed in the coarse-grained region. The procedure for obtaining the optimal summation rule is developed based on deriving and then fitting the atomic energy distribution within an element under the constraint of a given shape function. To validate the generalized framework, test problems including nonlocal harmonic and anharmonic models undergoing tensile, shear and bending deformations will be solved using linear, bilinear and quadratic elements, respectively. Results obtained using the proposed optimal summation rules for the different element types will be compared with Gauss quadrature for accuracy. Through error structure analyses, it is found that the proposed summation rule always outperforms Gauss quadrature, even when the later employs more quadrature points than the former. It is argued that widely-used numerical quadrature techniques such as Gauss quadrature are not optimal for coarse-grained atomic energy approximation because they do not account for the discrete nature of the atoms. In contrast, the present summation rule is derived consistently from the underlying atomic energy distribution, and thus has better accuracy and smaller computational cost.
\end{abstract}

\section{REFERENCE}

[1] Yang, Q., Biyikli, E., To, A.C. Comput. Methods Appl. Mech. Eng. 2013, 258, 26-38. 